\title{
A window to the autism: the political role of the difference of an objectile in the homogeneous school
}

\section{SIGRADI2018 TECHNOPOLITICAS xxii congresso da sociedade iberoamericana de gráfica digital 22th conference of the iberoamerican society of digital graphics 07|08|09|novembro|2018 iauusp | são carlos | spbr}

\author{
Prof. Dr. Rovenir Bertola Duarte \\ Universidade Estadual de Londrina | Brazil | rovenir@uel.br
}

\author{
Arq. Ayla Ziger Dalgallo \\ Universidade Estadual de Londrina | Brazil | ayla.ziger@gmail.com
}

Disc. Maria Luisa Consalter Diniz

Universidade Estadual de Londrina | Brazil | maria.luisa.consalter@gmail.com

Disc. Thais Romão Magoga

Universidade Estadual de Londrina | Brazil | thaisromaomagoga@gmail.com

\begin{abstract}
This paper approaches the insertion of an objectile in the homogeneous space of a school, looking to bring flexibility and responsiveness to assist a user with Autism Spectrum Disorder (ASD). The research concerns with photosensitivity, a problem faced by almost $25 \%$ of the children with autism (Miller-Horn; Spence; Takeoka, 2011). The study is based on the theories for ASD environments that speak of 'sensorial perception' and 'thinking with imagery' (Mostafa, 2008), and the coexistence of Sensory Design Theory and Neuro-Typical Method (Pomana, 2015). The result consists of a gadget developed in MIT App Inventor tool and a curtain that interact responsively through an Arduino code, for a new connection between the user and his surroundings.
\end{abstract}

Keywords:Objectile; Responsive Architecture; Architecture and autism; ASD; Inclusive school.

\section{INTRODUÇÃO}

O ambiente escolar é marcado pela classificação e separação de pessoas, de modo a produzir classes tão uniformes quanto necessárias para o ensino simultâneo. Esta uniformização transborda para o espaço arquitetônico produzindo homogeneidade, algo que pode ser visualizado nos enfileiramentos de blocos, salas, carteiras, portas e janelas idênticos (Figura 1). Contudo, este ambiente homogêneo é ocupado por alunos com as mais diferentes demandas, podendo estar entre eles um portador do Transtorno do Espectro Autista (TEA). A presença deste aluno com TEA e da diferença que sua presença traz tornou-se mais concreta no Brasil após a promulgação da Lei Berenice Piana (Lei no 12.764/2012). O trabalho parte da questão de como tratar tecnologicamente a diferença num ambiente moldado homogeneamente. Para colaborar com esta questão propõe-se a troca de um objeto fixo por outro variável, ou seja, um objectile (Duarte; Sanches; Lepri, 2017).

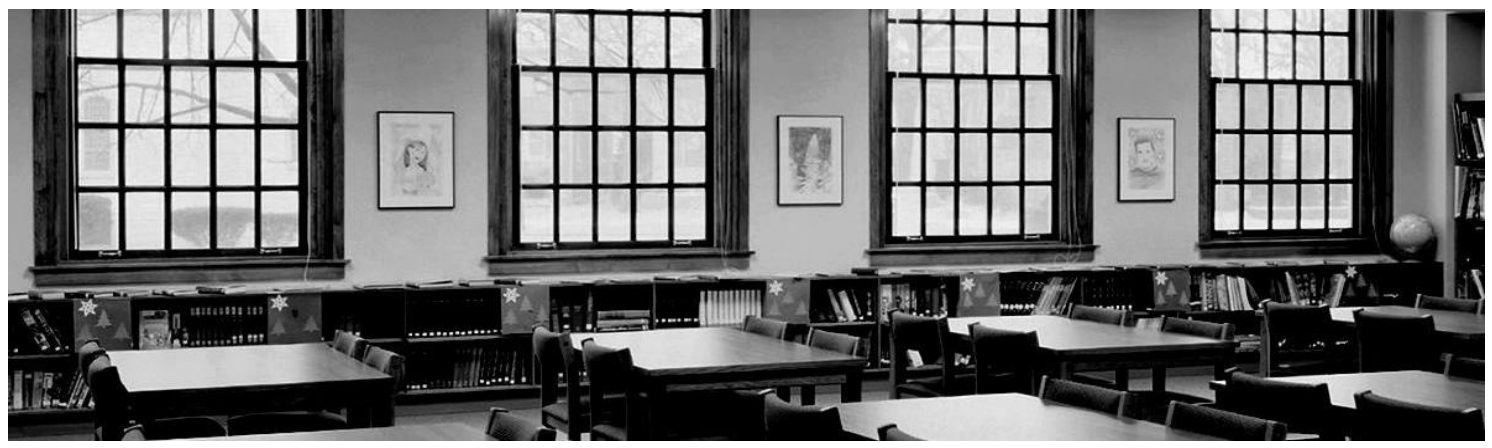

Figura 1: Um típico ambiente homogêneo escolar, baseado na repetição de carteiras, cadeiras e janelas. Fonte: Autores.

Este artigo relata uma atividade de iniciação científica abrigada em uma pesquisa teórica maior sobre objectiles (2015-2017), e encampada por parte do grupo dessa pesquisa. Esta atividade inicia com a reflexão teórica proposta nessa pesquisa sobre o emprego de objetos variáveis na arquitetura, propondo substituir um dos elementos homogêneos e estático da sala de aula por outro variável. Em lugar de pensar o espaço pela ótica das massas, pautado na fabricação em série, propõe-se pensar na "flutuação dos comportamentos individuais" (Cache, 1995, p.115). O resultado é um exercício exploratório que, ainda sem uma avaliação empírica para sua comprovação, sugere modos de pensar e projetar o espaço de maneira a contemplar a diferença.

A homogeneidade da escola é construída pela média de suas necessidades, desconsiderando os casos muito diferentes. Entretanto, para o aluno com TEA um ambiente com algum barulho e agitação, comuns para a média, pode interferir diretamente em seu comportamento. Pesquisas atuais (Miller-Horn, Spence e Takeoka, 2011) apontam que próximo a $25 \%$ das crianças autistas podem apresentar fotossensibilidade, 
podendo ser incomodadas por uma entrada de luz atuando como um gatilho para crises. Neste sentido caberia repensar a janela repetida serialmente na escola homogênea, pois surge a necessidade de inserir a diferença para gerar um ambiente amigável à criança com TEA, com controle das "entradas sensoriais" (Mostafa, 2008). Através de um objectile, um objeto tecnológico variável, propõe-se ajudar a produzir um espaço mais sensível a diversidade, uma arquitetura mais responsiva com relações enriquecedoras entre pessoas e ambiente (Beesley; Khan, 2009).

A pesquisa dedicou-se na elaboração de um objectile para a janela da sala de aula, visando controlar a entrada de luz e deixar o ambiente escolar menos hostil a uma criança com TEA. O dispositivo foi pensado para crianças que possam frequentar o ensino regular, em uma faixa etária entre 3 e 7 anos. A escolha dessa faixa se explica pelo desenvolvimento psicomotor que acontece neste período. Oliveira (1997) observa que as crianças nesta idade começam a aprimorar suas habilidades e refinar seus movimentos, conquistando um maior domínio de suas ações dentro de um espaço e tempo determinados.

\section{O AMBIENTE ESCOLAR HOMOGÊNEO E A INCLUSÃO DA DIFERENÇA}

Silva (2014) afirma que a escola moderna tem a homogeneização como marca de sua racionalidade. $\mathrm{Na}$ busca de criar condições para o ensino simultâneo, a escola atual revela um desejo por classificação, normalização e uniformização (por idade, capacidade de aprendizado, gênero, etc.). Essa homogeneização, dividida em classes e séries, visa controle e disciplina em prol de produzir um ambiente voltado para o ensino simultâneo e produtivo. Nas palavras de Brighente e Mesquida, "...tudo visando à obediência e a economia de tempo (...) parece haver um sentido econômico e produtivo, objetivando tornar os corpos continuamente homogêneos" (2011, p.2399). Essas reflexões claramente se inspiram no pensamento de Foucault (1999), na ideia de uma máquina de ordem e regularidade que sujeita os corpos a movimentos regulares, visando excluir agitação, distração para impor uma hierarquia e vigilância. Em prol dessa homogeneidade, como também pela fabricação de "corpos dóceis", a escola faz o necessário para dividir em iguais e obter o controle para o ensino simultâneo, contudo, como proceder quando se necessita incluir nesta homogeneidade a diferença?

\section{A ESCOLA INCLUSIVA E O ALUNO COM TEA}

Após a Constituição Federal de 1988, ficou assegurado o direito de atendimento aos portadores de deficiência nas escolas regulares. Com o Estatuto da Criança e do Adolescente (Lei 8.069/1990) este direito foi reforçado no artigo 54, onde se definiu a realização de atendimento educacional especializado. No caso dos portadores de TEA esse direito foi assegurado definitivamente em 2012 com a Lei Berenice Piana 12.764, que institui a Política Nacional de proteção dos direitos da pessoa com TEA. Assim, a escola pensada homogeneamente precisa de um salto qualitativo para a inclusão da diferença, reconhecendo cada indivíduo: uma escola inclusiva pautada no direito à diversidade.
A Declaração de Salamanca de 1994 aborda o princípio fundamental da educação inclusiva, por uma escola onde todas as crianças devem aprender juntas, independentemente das dificuldades e diferenças. Como defende Silva (2011), a educação inclusiva propõe um ensino em que a escola se adapta ao indivíduo que ela busca incluir, e não o contrário. "As escolas inclusivas devem reconhecer e responder às necessidades diversas de seus alunos" (Declaração de Salamanca, 1994, p.11). A proposta é não excluir em nome da normalidade padronizada. Incluir definiria aceitar incondicionalmente as diferenças dos outros e, como explica Cunha (2014), não se pode pensar em inclusão escolar sem pensar em um ambiente inclusivo. Assim, persiste a questão: Como pensar a inclusão da diferença em um ambiente marcado pela homogeneidade? Como contribuir com o espaço arquitetônico escolar para atender alunos com TEA?

\section{AS CARACTERÍSTICAS PERCEPTIVAS DO PORTADOR DE TEA E UM ESPAÇO ARQUITETÔNICO PARA AUTISTAS}

Os alunos portadores de TEA possuem comportamentos e dificuldades muito diferentes. Como explica Kanner (1943), eles podem apresentar dificuldades na interação social, comunicação e interesse seletivo. Baron-Cohen et al. (2009) observam, em sua pesquisa sobre autistas em escolas, um comportamento fortemente repetitivo, desatenção ou dificuldade de foco, como também o atraso no aprendizado da linguagem. Silva (2011) destaca que seus comportamentos podem revelar estereotipias, hiperatividade, agressividade e desinteresse pelas atividades escolares.

Especialmente sobre a percepção espacial, Mostafa (2008), arquiteta especialista em ambientes para autismo, explica que 0 aluno autista identifica 0 ambiente arquitetônico mais ao redor de um zoneamento sensorial que de um zoneamento funcional convencional. Para ela, os alunos com TEA possuem uma espécie de mau funcionamento sensorial na assimilação de informação em ambientes muito estimulantes. Pomana (2015), pesquisador em arquitetura para autistas, lembra que cerca de $85 \%$ dos autistas percebem cores com uma intensidade maior, muitas vezes com alta vibração e tons fluorescentes. Assim a neutralidade nos ambientes é bem-vinda, mas mesmo assim, Mostafa (2008) acredita que o comportamento deve ser afetado por um ambiente sensorial cuidadosamente projetado, devendo tratar de aspectos como cores, texturas, iluminação e sensação de fechamento. Sobre esses tratamentos do espaço, Pomana (2015) vê uma espécie de oposição entre duas abordagens: por um lado, sugerir o isolamento da estimulação sensorial (Sensory Design Theory), por outro, pensar sobre a necessidade de adaptação progressiva aos ambientes convencionais (Neuro-Typical Method). Segundo Pomana, as duas abordagens devem coexistir dentro do possível, pensando em como combiná-las, e neste sentido caminha este estudo.

Aqui começam os problemas entre o aluno autista e a sala de aula homogênea de plano aberto. Mostafa (2008) comenta que este tipo de espaço não é recomendado por estimular a confusão sensorial. A especialista observa a necessidade de se controlar a "entrada sensorial" (Mostafa, 2008) do ambiente para não produzir hiperestimulação. Um som ou movimento fora do controle da 
criança autista pode provocar distúrbios acentuados, de modo que a previsibilidade seja desejada. Como destaca Kanner (1943), crianças autistas revelam resistência a mudanças imprevistas e gostam de rotinas. Por essa razão, Mostafa (2008) recomenda a abordagem projetual de limitar 0 ambiente sensorial por meio de compartimentação, controlando distrações visuais e restringindo seus campos de visão periférica. Ela sugere pequenos compartimentos funcionais dentro da sala de aula para o controle da entrada sensorial, algo que chama de "espaço de fuga" (Mostafa, 2008). A compartimentação capitalizaria a atenção do autista, com a formação de um espaço protegido e seu. Porém, a própria Mostafa (2008) destaca a dificuldade de fazer esse tipo de espaço em ambiente público, como as escolas regulares, necessitando pensar em mecanismos que permitam a diferenciação do espaço. É um desafio criar um ambiente previsível, ao mesmo tempo em que permita abertura para o aprendizado de habilidades essenciais da vida cotidiana. Como Carvalho (2016) alerta, a inclusão do autista nas escolas é um dos grandes desafios da atualidade, há a necessidade de promover um ambiente que 0 aluno com TEA se mantenha tranqüilo e confortável, mas com estímulos suficientes para que não se sinta entediado.

\section{A QUESTÃO DA FOTOSSENSIBILIDADE: UM LUGAR PARA TUDO E TUDO EM SEU LUGAR}

Wing e Gould (1979) destacam a diversidade de comportamento dos autistas, um espectro muito amplo de sensibilidades e reações. Como Baron-Cohen et al. (2009) observam, qualquer ruído ou facho de luz diferenciado pode atuar como um gatilho para crises. A hipersensibilidade sensorial é frequentemente diagnosticada nas crianças com TEA, sendo a fotossensibilidade uma de suas formas mais comuns. Humphreys (2011) diz que ao se pensar a arquitetura de acordo com as necessidades do autista, deve-se dar uma atenção especial à fotossensibilidade, afinal, uma variação luminosa muito grande pode criar situações que agem como estímulos visuais excessivos.

Em um estudo precursor desenvolvido no Boston Children's Hospital, ficou evidenciada a alta incidência de fotossensibilidade em crianças com TEA, aproximadamente $25 \%$, aumentando em casos de autistas epiléticos (Miller-Horn; Spence; Takeoka, 2011). Assim, a luz passando pela janela demanda um controle de "entrada sensorial", pois pode relacionar-se diretamente com o comportamento da criança autista na sala de aula. Neste sentido, a janela uniforme e padrão do ambiente homogêneo, como também sua luminosidade sem controle, entram em direto conflito com o espaço escolar inclusivo. Como explica Mostafa (2008), no caso de alunos com TEA é necessário que prevaleça a máxima: "um lugar para tudo e tudo em seu lugar". Neste sentido, cada espaço precisa ser pensado de modo a ser receptível para este aluno. Como explica Carvalho (2016), ainda que a presença do autista na escola regular seja uma vitória, "a escola é quem deveria se adaptar para recebê-lo” e não o contrário.

Nesta direção, a pesquisa propõe um dispositivo que possa quebrar com a excessiva homogeneidade da sala, que permita caracterizar o espaço do aluno com TEA e, ao mesmo tempo, que sirva de controle de entrada sensorial para a janela. Contudo, considerando a necessidade de trabalhar uma adaptação mais progressiva para as variações lumínicas (Neuro-Typical Method), como também, devido ao espectro muito amplo de comportamentos, precisa-se projetar uma arquitetura mais flexível e dinâmica. Como observa Pomana (2015), existe a necessidade de os ambientes serem flexíveis para acomodar muitas circunstâncias diferentes, pensando sempre em espaços de integração e convivência como um passo adiante. De que forma a arquitetura pode parecer confortante e estimulante para uma criança autista e, ainda, responder a situações além da normalidade do homogêneo? O que se propõe é incorporar à arquitetura algo que possa lhe atribuir flexibilidade, mas também, permita certa sensibilidade ao aluno autista, buscando um diálogo entre a criança com TEA e o ambiente que ela vive.

\section{UM OBJETO VARIÁVEL RESPONSIVO: UMA ALTERNATIVA À JANELA UNIFORME}

Um modo de buscar esse diálogo entre a criança com TEA e seu ambiente escolar pode ser através de dispositivos tecnológicos que busquem uma arquitetura responsiva e flexível. É importante ver a arquitetura por sua potência de desenvolver relações mais enriquecedoras entre pessoas e ambientes, um compromisso renovado por aproximações mais orgânicas e complexas entre os ambientes e seus ocupantes (Beesley; Khan, 2009). Pensar na produção de um dispositivo que não fosse indiferente as necessidades do aluno autista. Beesley e Khan (2009) destacam que o usuário poderia ver a arquitetura mais próxima, semelhante como vê um animal doméstico que, com alguma empatia, negociaria uma comunicação. Eles comentam sobre a horripilante ideia de o mundo ser indiferente a nós, de modo que acabamos buscando nas coisas algo como um "outro" que nos escuta, assim conversamos com animais e objetos, como um carro. A proposta seria introduzir uma interface de comunicação entre esse aluno e seu espaço, produzindo algo que Beesley e Khan (2009) chamam de "dança sofisticada", uma ligação entre o aluno e sua janela. Entre tantas janelas aquela, agora, seria diferente.

Para torná-la responsiva seria necessário também que esta adquirisse alguma capacidade de flexibilidade e variação. Como Kronenburg (2004) explica, a potência de um edifício responsivo está em sua flexibilidade, em construções projetadas para responder facilmente a mudanças ao longo do tempo. Para esse pesquisador, essa flexibilidade permite a arquitetura ajustar-se a sua finalidade e se acomodar à experiência e intervenção dos usuários, aproveitando-se mais rapidamente da inovação técnica (Kronenburg, 2004). Em busca desta arquitetura flexível e responsiva propõe-se a inclusão nesta janela de um objeto tecnológico variável, o chamado objectile. Termo criado pela parceria entre o filósofo Gilles Deleuze e o arquiteto Bernard Cache (a junção das palavras francesas object + projectile). O objectile trata de um objeto variável no qual a flutuação substitui a permanência da lei (Deleuze, 1991). Em outras palavras, a necessidade de tomar a variação como objeto, onde no lugar de um objeto-janela estático tratar-se-ia de uma família de variações e eventos: uma família enquadrada por parâmetros (Deleuze, 1991).

3 
Esse objectile pode ser pensado como uma infinita variedade de objetos em um só, todos distintos em alguns parâmetros e semelhantes em outros (Carpo, 2011). Um objeto que permita tal variabilidade necessária para flexibilizar os espaços, que possibilitem a incorporação das diferenças em um ambiente homogêneo (como as demandas do aluno com TEA dentro do ambiente escolar regular). Kronenburg (2004) considera a flexibilidade como algo essencial para o sucesso da arquitetura, afinal para ele, os seres humanos são criaturas flexíveis que manipulam os objetos, se movem e operam na busca de produzir lugares e ambiente mais acolhedores. No fim das contas, todos precisam de alguma maneira de um espaço mais seguro, que possa servir de "espaço de fuga" e tratar das entradas sensoriais. Kronenburg (2004) explica que alterar o ambiente para as suas próprias necessidades é uma característica comum ao ser humano que transforma um espaço anônimo em um "lugar" específico. Deste modo, a proposta é enxertar um objectile, e sua variabilidade, dentro do espaço homogeneizado da escola, procurando um modo do aluno com TEA construir um lugar mais acolhedor.

\section{DESCRIÇÃO DO OBJECTILE PARA JANELA}

O dispositivo proposto pela pesquisa trata-se de um objectile para controle das entradas sensoriais em uma janela convencional. Esse dispositivo funcionaria como o que Peeters (Apud Mostafa, 2008) chama de "pistas visuais", que poderia sinalizar uma área da sala como seu lugar ou ajudar a compor o seu "espaço de fuga", ao mesmo tempo, que controlaria a entrada de luz. Essas "pistas visuais" colaborariam com a identificação espacial mais visual que funcional dos alunos com TEA, como um poderoso efeito associativo e comunicativo. Segundo Mostafa (2008) as crianças com TEA pensam em imagens, onde padrões, cores ou abstrações podem ser usadas para comunicar às crianças o caráter das diferentes zonas. Por essa razão o dispositivo se divide em duas partes. A primeira trata-se de uma interface de comunicação com o usuário (input) através de um aplicativo para tablet ou celular. A segunda parte pode ser pensada como um mecanismo-objectile acoplado à janela que serviria para controlar a entrada de luz a partir dos sinais do primeiro (output). Este é composto pela programação em Arduino, um servomotor para rotacionar e o próprio objeto conectado à janela (Tabela 1).

Tabela1: Os componentes formadores do dispositivo: o aplicativo para tablet e o mecanismo-objectile para a janela

\begin{tabular}{lll}
\hline COMPONENTES & FUNÇÃO & INTERAÇÃO \\
\hline Aplicativo para tablet & $\begin{array}{l}\text { Receber o comando gerado pela } \\
\text { criança através do aplicativo } \\
\text { Captar sinal bluetooth e traduzir em }\end{array}$ & Tato (sentido) - software - bluetooth \\
ângulo de rotação para servomotor \\
Servomotor & $\begin{array}{l}\text { Rotacionar módulo cilíndrico de acordo } \\
\text { com comando recebido } \\
\text { Controle de iluminação através de }\end{array}$ & Servo (arduino) - pivô de rotação \\
torção dos módulos cilíndricos & Servomotor - visão (sentido) \\
\hline
\end{tabular}

\section{A INTERFACE DIGITAL DA JANELA NO TABLET}

Como comentado, os alunos portadores de TEA "pensam com imagens", sua orientação espacial faz-se mais através de suas relações perceptivas do que com zoneamentos funcionais (Mostafa, 2008). Assim, a primeira parte desse dispositivo busca explorar essas preferências, ao mesmo tempo em que visa desenvolver atividades motoras relativas à sua idade. O exercício de design deste aplicativo inicia elencando ações consideradas possíveis para a faixa etária a ser trabalhada: apertar, mover, girar, colocar e tirar. Por outro lado, como balancear estímulos para obter a resposta da interface, produzindo um sistema mais intuitivo, tais como sons, imagens, vídeos, luminosidade, temperatura, textura, odores e cores sem excessos? Após considerar o amplo espectro de diferenças entre alunos com TEA, optou-se por movimentos motores mais comuns, como "movimentar e arrastar", para não gerar estresses para crianças com maior dificuldade de coordenação fina. Essa interface enviaria o sinal do movimento da criança para o dispositivo acoplado à janela, para ativar os cilindros da cortina e controlar a entrada de luz.

A ponte entre a criança e a janela seria um jogo em forma de aplicativo que relaciona a mudança de posição de elementos na tela do dispositivo móvel com a rotação dos cilindros da cortina. A programação do seu protótipo foi realizada na plataforma MIT App Inventor@ e conectados via bluetooth ou módulo WI-FI (Esp8266) com o dispositivo encaixado na janela e suas cortinas. Nessa plataforma, a linguagem $\mathrm{C}++$ é programada em blocos, numa interface direta com o layout da tela do celular. Primeiramente, incorporaram-se os comandos necessários para estabelecer uma conexão via Bluetooth ou módulo WI-FI com o sistema Arduino. Feito isso, foram inseridos List Pickers, que possibilitam a interação do usuário com o tablet para a efetuar. Após estabelecer um meio de enviar dados pelo aplicativo, cinco sliders foram incorporados ao algoritmo, cada um responsável pela rotação de seu respectivo motor servo. Os sliders são controlados pelo simples deslizar de dedos e a posição em que o mesmo se encontra é lida pelo algoritmo e, então, transformada em um número Natural entre 0 e 180 graus. Este número, por sua vez, é retornado pelo mesmo e enviado via Bluetooth ou módulo WI-FI para a placa do sistema Arduino, onde é lido pelo seu código como o ângulo de rotação do motor servo.

Para funcionar de modo mais atrativo, a interface foi pensada para funcionar como um jogo que a criança poderia manusear. Um dos desenhos pensados para 0 jogo faz uso de um círculo com diferentes desenhos representando as fases da lua (Figura 2). Esses desenhos seriam modificados de acordo com a movimentação realizada pela criança, de maneira que a lua se manifestaria de algum modo, por exemplo, 
adquirindo mais ou menos brilho conforme esta fosse conectada com algumas estrelas. O objetivo do jogo seria conectar cinco estrelas à lua, a cada estrela conectada, a lua fica mais iluminada sugerindo mais giro nas cortinas. A intensidade da iluminação dependeria da localização da estrela conectada, quanto mais distante, maior a iluminação da lua. O design da interface necessitaria buscar simplicidade para não produzir excesso de estímulos, evitando a introdução de muitas cores, intensidades e formas. Ao término das 5 ligações com as estrelas, a imagem da lua resultante apareceria na tela. Ao enviar esse resultado para o software no Arduino, acontecem os giros do dispositivo acoplado à janela da sala de aula.

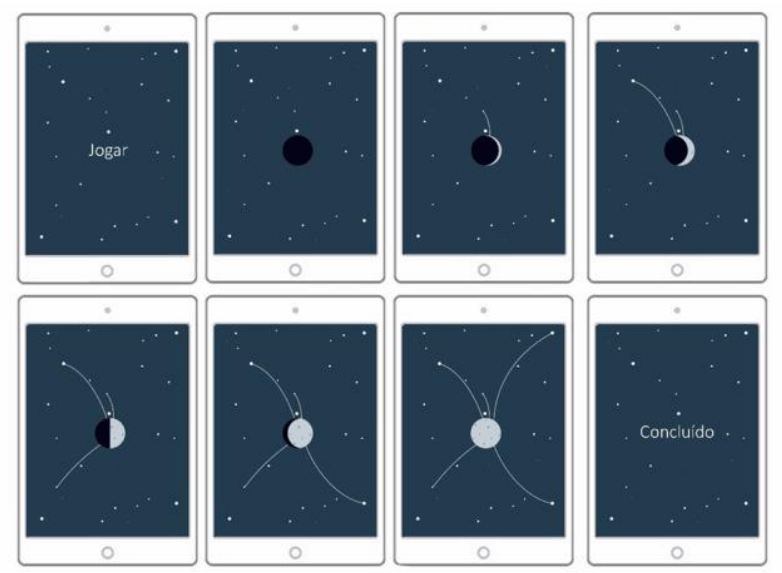

Figura 2: A interface desenhada com a figura de lua ao centro e a simulação de deslocamento das estrelas. Fonte: Autores.

\section{O DISPOSITIVO RESPONSIVO DA JANELA: \\ OBJECTILE}

A segunda parte do dispositivo dedica-se a elaboração de um mecanismo que, provocado pelo sinal do aplicativo, controla a entrada de luz da janela. Esse objeto variável funcionará como uma espécie de "maquiagem sensorial" (Mostafa, 2008) que poderá personalizar as características perceptivas de uma zona da sala, voltando para cada especificidade de cada aluno. A proposta consiste em produzir um aparato que encaixaria na janela pela parte de dentro da sala de aula, composto por módulos verticais que giram sobre si mesmos. Buscou-se evitar o conflito entre seu desenho e o uso pelos alunos com TEA, fazendo uso de formas arredondadas, materiais não agressivos e uma movimentação suave. Deste modo, esses módulos foram pensados como cilindros, cujos corpos são tubos têxteis conectados em suas bases por um material rígido circular. A base inferior de cada cilindro é fixada à estrutura do objeto, enquanto a superior faz conexão com um motor que gira até $180^{\circ}$. A cada giro, formam-se estrangulamentos no corpo do cilindro permitindo maior entrada de luz. Os módulos cilíndricos trabalham de forma independente, permitindo maior controle e desenho na forma como se protege a passagem de luz. Quando a rotação da base superior do cilindro for igual a zero, o objeto apresenta-se totalmente opaco, de modo a permitir apenas a passagem de iluminação filtrada, dependendo da característica do tecido utilizado no seu fechamento. Ao rotacionar a base superior, permite-se a entrada de luz direta ou difusa, conferindo à criança o poder de controlar os níveis de iluminação natural (Figura 3).

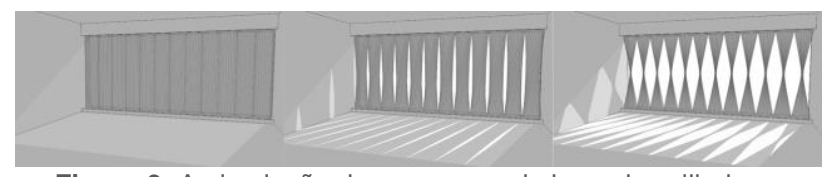

Figura 3: A simulação da passagem de luz pelos cilindros. Fonte: Autores.

No exercício proposto para simulação foi desenvolvido um modelo na escala 1:10, com módulos feitos em papel e tecido (Figura 4). As bases superiores de cada cilindro estavam conectadas a um servo motor SG90 (um servo por módulo) e controladas por sistema Arduino. No primeiro momento, os dados de entrada do modelo eram dados por potenciômetros (um potenciômetro por servo), que aplicavam proporcionalmente 0 seu ângulo de rotação ao do servo motor ao qual estava relacionado.

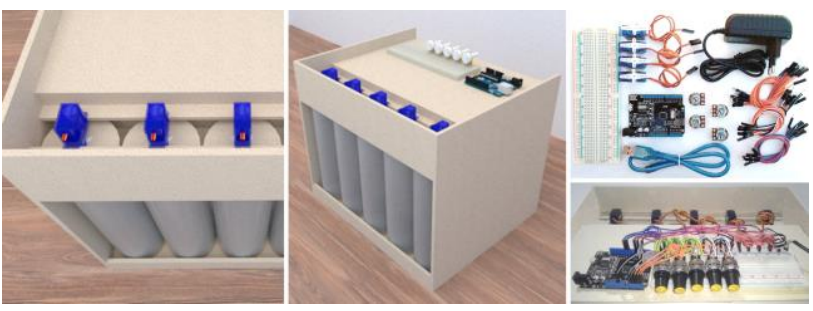

Figura 4: O protótipo em escala 1:10 do dispositivo. Fonte: Autores.

Posteriormente, com a incorporação do aplicativo para tablet, o input passou a ser fornecido pelo jogo, bastando substituir os potenciômetros utilizados anteriormente por um módulo receptor de bluetooth ou WI-FI. Também foi necessário adaptar a escala de conversão dos dados input e output. A programação final do Arduino foi bastante simples (Figura 5), apenas recebendo o sinal gerado pelo aplicativo do tablet e traduzindo em ângulos para rotação de cada servo.

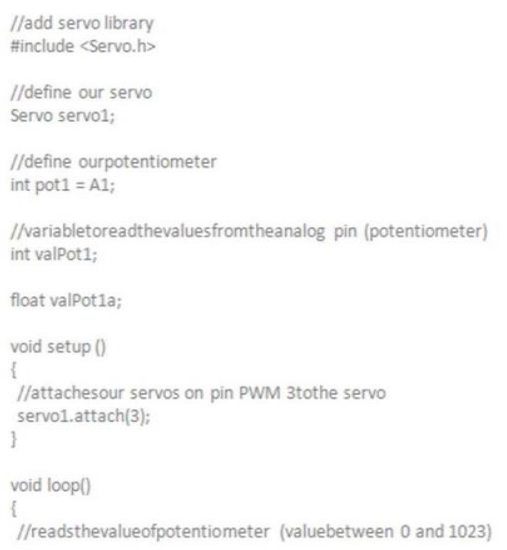

valpot1 = analogRead(pot1);

valPot1a = map (valPot1, $0,1023,0,179$ ); ///scale it to use it withthe servo (valuebetween 0 and 180 ) servo1.write(valpot1a); //set the servo position accordingtothescaledvalue delay(15);

Figura5: A descrição código base do Arduino. Fonte: Autores.

Nesse código, após identificar os servos e definir os potenciômetros, foi delimitado o intervalo de valores variáveis, lido através da rotação dos potenciômetros. Em seguida, foi inserida a função setup responsável por vincular cada servo com o seu respectivo pin. Somente então o loop do código pode ser definido: inicialmente o Arduino realiza a leitura do input, fornecido pelos potenciômetros (valor entre 0 e 1023) e em seguida converte este número para equivaler aos graus de rotação do servo motor (entre 0 e 180). Após esses 
passos é fornecido o output, que rotaciona o servo motor correspondente ao número gerado pela conversão.

\section{RESULTADOS}

O tema da fotossensibilidade em arquitetura para autistas até o momento é pouco estudado, ainda mais quando se fala em sala de aula no ensino regular. A pesquisa buscou responder às condições apontadas por estudiosos em espaços para TEA, atentando para as questões como "controle sensorial" e "pensando com imagens" (MOSTAFA, 2008), ao mesmo tempo em que considerou a coexistência das abordagens Sensory Design Theory e Neuro-Typical Method (Pomana, 2015). Assim, através de um aparato técnico, que aproxima a visão e o tato do jogo no tablet com a observação do que ocorre em sala de aula em relação à luz, pode-se pensar em uma colaboração com um aprendizado ambiental. Deste modo, a sala homogênea, que ensinaria basicamente disciplina, ganha nuances mais particulares e inclusivas. Infelizmente pelo prazo da pesquisa não foi possível avaliações em sala de aula, não podendo medir algumas conseqüências importantes tais como: a capacidade do dispositivo em produzir integração e sociabilidade entre os alunos com TEA e os demais alunos; a possibilidade de uso deste dispositivo no espaço aberto da sala de aula além dos "espaços de fuga"; e como o estímulo das sombras projetadas no chão poderia afetar o comportamento das crianças com TEA.

De todo modo, é muito provável, segundo relatos de Mostafa (2008), que este dispositivo não seja suficiente para produzir um "espaço de fuga" seguro ao aluno com TEA, necessitando outros tipos de adaptações. Esse aparato está mais próximo da formação do zoneamento sensorial em lugar do funcional. O conjunto responsivo, aplicativo no tablet e objectile na janela, gera dentro da sala de aula um ambiente mais participativo e experimental, buscando uma construção de lugar a partir da forma que o espaço responde ao desejo do usuário. Também se deve destacar o amplo espectro de comportamentos de crianças com TEA, entendendo que muitas situações são difíceis de prever e que dependeriam bastante da intervenção do professor em sala de aula. Não conseguindo precisar a reação de cada criança na manipulação do aplicativo, necessitando talvez mais opções de interfaces. Espera-se continuar a pesquisa com avaliações com simulação, entretanto, foi possível observar a potência que tem um objeto variável (objectile) na transformação do espaço pensado para massa, tornando-o um espaço mais sensível.

\section{REFERÊNCIAS}

Baron-Cohen, S. et al. (2009). Autism spectrum prevalence: a school-based UK population study. British Journal of Psychiatry, 194, 500-509.

Beesley, P.; Khan, O. (eds.) (2009). Responsive Architecture/Performing Instruments. New York: The Architectural League of New York. Print.

Brasil (1990). Estatuto da criança e do adolescente. Estatuto da criança e do adolescente: Lei n. 8.069, de 13 de julho de 1990, Lei n. 8.242, de 12 de outubro de 1990. (3rd ed.) Brasília: Câmara dos Deputados, Coordenação de Publicações.

(2012). Lei no 12.764, de 27 de dezembro de 2012. Institui a Política Nacional de Proteção dos Direitos da Pessoa com
Transtorno do Espectro Autista; e altera o § 3odo art. 98 da Lei no 8.112, de 11 de dezembro de 1990. Retrieved from: <http://www.planalto.gov.br/ccivil_03/_ato2011-

2014/2012/lei//12764.htm>. Acesso em: 17 nov. 2015.

Brighente, M. F., Mesquida, P. (2011, November). Michel Foucault: corpos dóceis e disciplinados nas instituições escolares. X Congresso Nacional de educação EDUCERE.Proceedings...Curitiba,PUC. Retrieved from: http://educere.bruc.com.br/CD2011/pdf/4342_2638.pdf

Cache, B. (1995). Earth Moves: The Furnishing of Territories. Massachusetts: Massachusetts Institute of Technology.

Carpo, M. (2011). The alphabet and the algorithm. Cambridge, Massachusetts: MIT Press.

Carvalho, J. L. (2016). Inclusão dos autistas nas escolas. Psicologia do Brasil (Site). Retrievedfromhttps://www.psicologiasdobrasil.com.br/inclusa o-dos-autistas-nas-escolas/

Cunha, E. (2014). Autismo e inclusão: psicopedagogia práticas educativas na escola e na família. (5th ed). RJ: Wak Ed., 2014.

Deleuze, G. (1991). A dobra: Leibniz e o barroco. Campinas: Papirus.

Duarte, R.B., Sanches, M. M.; Lepri, L.. (2017) Objectile e as "novas pretensões" do projeto paramétrico em arquitetura. Revista Gestão e Tecnologia de Projetos. USP-IAU, 12 (3), 59-75.

Humphreys, S. (2011, June). Autism and Architecture. AutismEurope's LINK magazine, 55, 9-13. Retrieved from http://www.autismeurope.org/publications/magazine-link.

Kronenburg, R. H. (2015). Flexible Architecture: Continuous and Developing. In B. Kolarevic, B. \& Parlac, V. (Eds.), Building Dynamics: Exploring Architecture of Change (p. 29-42). New York: Routledge.

Miller-Horn, J.; Spence, S., Takeoka, M. (2011, December 2 6).Photoparoxysmal responses in children with autism spectrum disorders. (Abstract 3.109). AES 65th Annual Meeting, Baltimore, MD, USA, 12 (01). Retrieved from https://www.aesnet.org/sites/default/files/file_attach/Professio nalEducation/Currents/2012/AbstSupVol12Num1/2011abstra cts_supplement1.pdf

Mostafa, M. (2008, March) An Architecture for Autism: Concepts of Design Intervention for the Autistic User. Archnet-IJAR, International Journal of Architectural Research, 2 (1), 189211.

Oliveira, G. D. C (1997). Psicomotricidade: educação e reeducação num enfoque psicopedagógico. Petrópolis, Rio de Janeiro: Vozes.

Organización de las Naciones Unidas para la Educación, la Ciencia y la Cultura (2014). Declaración de Salamanca y Masco de acción. Ministerio de Educación y Ciencia de España. UNESCO. Retrieved from http://www.unesco.org/education/pdf/SALAMA_S.PDF.

Pomana, A. (2015, March). Architecture for autism: improving designs for autistic integration. ICAR International Conference on Architectural Research, Proceedings... Bucharest.

Silva, C. R. C. da (2014, October). Formação de classes homogêneas: condição para uma aprendizagem mais regular em menor tempo? X ANPED Sul, UDESC,Proceedings... Florianópolis, Brazil. Retrieved from http://xanpedsul.faed.udesc.br/arq_pdf/933-0.pdf

Wing, L.; Gould, J.(1979, March). Severe impairments of social interaction and associated abnormalities in children: Epidemiology and classification. Journal of Autism and Developmental Disorders, 9(1), 11-29. 